Among the orbits produced by a central force varying as the nth power of the radius vector are included the isogonal trajectories of the curves

$$
y^{\prime}=-\tan \frac{1}{2}(n+1) \theta .
$$

This construction yields in the case $n=-2$ (Newtonian law) parabolas with focus at the origin ; in the case $n=1$ (elastic law) equilateral hyperbolas with center at the origin; in the case $n=-5$ the circles through the origin; and in the case $n=-3$ equiangular spirals with pole at the origin.

Columbia University.

\title{
ON THE EQUATIONS OF QUARTIC SURFACES IN TERMS OF QUADRATIC FORMS.
}

\author{
BY DR. C. H. SISAM.
}

(Read before the American Mathematical Society, September 5, 1907.)

THE quartic surfaces whose equations are of the form

$$
\phi_{2}(A, B, C, D)=0,
$$

where $\phi_{2}, A, B, C$ and $D$ are quaternary quadratic forms, were the subject of a paper by $\mathrm{H}$. Durrande in the Nouvelles Annales.* By counting the number of constants involved, Durrande concluded that the most general quartic surface could be represented by an equation of the above form. He recognized, however, that his reasoning was not rigorous.

It will here be shown that the coefficients of the quartic surface determined by this equation are not independent, but are subject to a single condition. It will also be shown that the equation of a general quartic surface can be written in the form

$$
\theta_{2}(A, B, C, D, E)=0,
$$

where $\theta_{2}$ is a quinary quadratic form.

Let $\phi_{2}=0$ be reduced to the form

$$
A^{2}+B^{2}+C^{2}+D^{2}=0
$$

where

$$
A \equiv \Sigma a_{i j} x_{i} x_{j} \quad(i \leqq j \leqq 4)
$$

\footnotetext{
* Durrande, Nouvelles Annales, ser. 2, vol. 9, p. 410.
} 
with similar expressions for $B, C$, and $D$. Let also

$$
\Sigma F_{i j k l} x_{i} x_{j} x_{k} x_{l}=0 \quad(i \leqq j \leqq k \leqq l \leqq 4)
$$

be the equation of the same surface in the ordinary form.

Equating the coefficients of like terms in the two equations of the surface, we obtain the following thirty-five equations :

$$
\begin{aligned}
& \Sigma a_{i i}^{2}=F_{i i i} \quad(i=1,2,3,4), \\
& \Sigma 2\left(a_{i i} a_{i j}\right)=F_{i i i j} \quad(i, j=1,2,3,4 ; i \neq j), \\
& \Sigma\left(2 a_{i j} a_{j j}+a_{i j}^{2}\right)=F_{i j j} \quad(i, j=1,2,3,4 ; i \neq j), \\
& \Sigma\left(2 a_{i i} a_{j k}+2 a_{i j} a_{i k}\right)=F_{i j j_{k}} \quad(i, j, k=1,2,3,4 ; i \neq j \neq k) \text {, } \\
& \Sigma 2\left(a_{12} a_{34}+a_{13} a_{24}+a_{14} a_{23}\right)=F_{1234},
\end{aligned}
$$

the summation extending over the quantities $b, c$, and $d$.

The necessary and sufficient condition that (1) be the equation of a general quartic surface is that these quantities $F_{i j k l}$ be independent functions of $a_{i j}, b_{i j}$, etc. They will be independent functions of these quantities if, and only if, at least one determinant of order thirty-five of the Jacobian matrix of thirty-five rows and forty columns

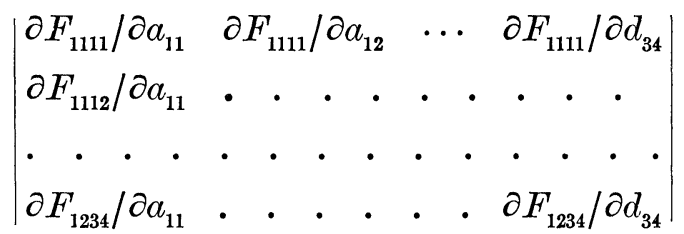

be different from zero.

If any of these determinants is different from zero, it will be impossible to find thirty-five quantities, $y_{1}, y_{2}, \cdots, y_{35}$, satisfying simultaneously the forty equations

$$
\begin{gathered}
\frac{\partial F_{1111}}{\partial a_{11}} \cdot y_{1}+\frac{\partial F_{1112}}{\partial a_{11}} y_{2}+\cdots+\frac{\partial F_{1234}}{\partial a_{11}} \cdot y_{35}=0, \\
\cdot \cdot \cdot \cdot \cdot \cdot \cdot \cdot \cdot \cdot \cdot \cdot \cdot \cdot \cdot \cdot \cdot \cdot \cdot \cdot \cdot \cdot \cdot \frac{\partial F_{1234}}{\partial d_{34}} \cdot y_{35}=0 .
\end{gathered}
$$

Consider, now, the system of quadrics 


$$
a A+\beta B+\gamma C+\delta D=0 .
$$

In this system there are, in general, ten * which break up into pairs of planes. Let the coordinate planes $x_{1}=0$ and $x_{2}=0$ be taken to coincide with one of these pairs of planes and $x_{3}=0, x_{4}=0$ with another pair. We may then, in the above forty equations, put $c_{12}=1$ and the remaining $c_{i j}=0$, also $d_{34}=1$ and the remaining $d_{i j}=0$.

It is now necessary that $y_{26}=y_{31}=y_{27}=y_{34}=y_{22}=y_{5}=y_{25}$ $=y_{8}=y_{28}=y_{29}=y_{13}=y_{32}=y_{16}=y_{17}=y_{35}=y_{23}=y_{30}=y_{24}$ $=y_{33}=0$. Twenty-four of the equations are therefore satisfied. In order that the other sixteen be satisfied by quantities $y$ not all zero, it is necessary and sufficient that the determinant

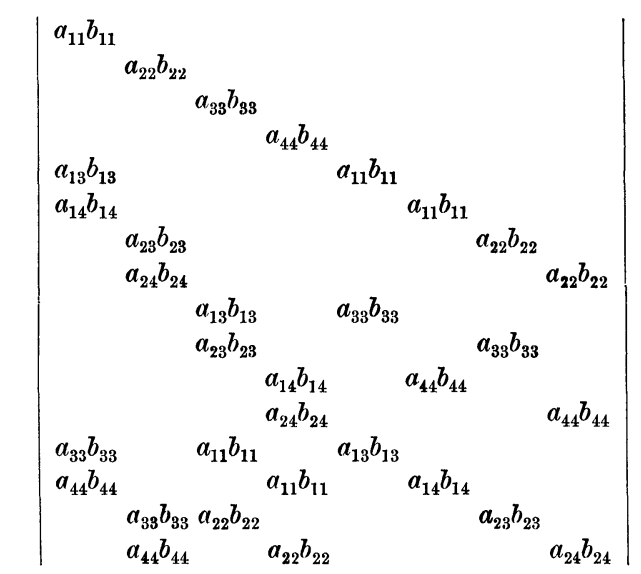

be equal to zero. Denote the columns of this determinant in order by (1), (2), etc. Replace the first column by $b_{11}(1)-a_{11}$ $(2)+b_{22}(3)-a_{22}(4)+b_{33}(5)-a_{33}(6)+b_{44}(7)-a_{44}(8)$ $+b_{13}(9)-a_{13}(10)+b_{14}(11)-a_{14}(12)+b_{23}(13)-a_{23}(14)$ $+b_{24}(15)-a_{24}(16)$. The first column so obtained vanishes identically. The determinant therefore vanishes identically and the $F_{i j k l}$ are not independent. The surface $\phi_{2}(A, B, C, D)=0$ is not, therefore, the most general quartic.

In order that the equation of a quartic may be put in the above form, only a single condition must be satisfied by the coefficients. For if we omit $F_{1234}$, and consider the remaining $F_{i j k l}$ as functions of the $a_{i j}$, etc., the Jacobian matrix does not vanish iden-

* Reye, Crelle, vol. 82 , p. 76 ; vol. 86, p. 89. 
tically. This may be seen by substituting the particular values $a_{11}=b_{22}=c_{33}=d_{44}=1$, the remaining $a_{i j}$, etc., being zero. Hence these quantities $F_{i j k l}$ are independent.

Finally, consider the quartic

$$
\theta_{2}(A, B, C, D, E)=0 .
$$

Let this equation be reduced to

$$
A^{2}+B^{2}+C^{2}+D^{2}+E^{2}=0 .
$$

Equate the coefficients of the terms of this equation to the corresponding terms of

$$
\Sigma k_{i j k l} x_{i} x_{j} x_{k} x_{l}=0 \quad(i \leqq j \leqq k \leqq l \leqq 4)
$$

and determine the jacobian matrix as before. That the determinants of this matrix do not all vanish identically is seen by taking for $A, B$, etc., the particular expressions

$$
A \equiv x_{1}^{2}, B \equiv x_{2}^{2}, C \equiv x_{3}^{2}, D \equiv x_{4}^{2}, E \equiv x_{1} x_{2} .
$$

Since these determinants do not vanish identically the $k_{i j k l}$ are independent. Hence the equation of an arbitrary quartic surface can be put into the form

$$
A^{2}+B^{2}+C^{2}+D^{2}+E^{2}=0 .
$$

URBANA, ILL.,

September, 1907.

\section{SYMBOLIC LOGIC.}

L'Algèbre de la Logique. Par Louis Couturat. Collection Scientia, No. 24. Gauthier-Villars, Paris, 1905. 100 pp. Symbolic Logic and its Applications. By Hugh MacColl. Longmans, Green, and Co., London, $1906 . \quad x i+141$ pp.

The Development of Symbolic Logic; a Critical-Historical Study of the Logical Calculus. By A. T. Shearman. Williams and Norgate, London, 1906. xi $+242 \mathrm{pp}$.

Symbolic logic is in the interesting though somewhat precarious state of being little known, less used, and much scorned by the majority of mathematicians and philosophers, for whom it might supposedly offer a region of intimate contact and 